\title{
Comment on: Cloning and characterization of porcine aquaporin 1 water channel expressed extensively in the gastrointestinal system
}

\author{
Ali Mobasheri
}

\author{
Ali Mobasheri, Molecular Pathogenesis and Connective Tissue \\ Research Groups, Department of Veterinary Preclinical Sciences, \\ Faculty of Veterinary Science, University of Liverpool, Liverpool, \\ United Kingdom \\ Correspondence to: Dr. Ali Mobasheri, Molecular Pathogenesis \\ and Connective Tissue Research Groups, Department of \\ Veterinary Preclinical Sciences, Faculty of Veterinary Science, \\ University of Liverpool, Liverpool, \\ United Kingdom. a.mobasheri@liverpool.ac.uk \\ Telephone: +44-151-7944284 Fax: +44-151-7944243 \\ Received: $2006-03-24$ \\ Accepted: 2006-04-21
}

(c) 2006 The WJG Press. All rights reserved.

Mobasheri A. Comment on: Cloning and characterization of porcine aquaporin 1 water channel expressed extensively in the gastrointestinal system. World J Gastroenterol 2006; 12(27): 4437-4439

http://www.wjgnet.com/1007-9327/12/4437.asp

\section{TO THE EDITOR}

Sir, I read with great interest the recently published article in the World Journal of Gastroenterology by Jin and co-workers ${ }^{[1]}$ on the cloning and characterization of porcine aquaporin 1 water channel from the pig liver and studies on its expression in the porcine gastrointestinal system. The authors should be congratulated for making this important and valuable contribution to the field of aquaporin biology and porcine gastrointestinal physiology. However, there are a number of unresolved issues and controversies concerning the expression of aquaporins (especially aquaporin 1) in the gastrointestinal system that are worthy of additional comment and discussion by Jin and co-workers.

It is now well established that aquaporin (AQP) water channels are a family of membrane proteins that facilitate water movement across cell membranes in plants and animals. Thus far, at least 13 human aquaporins (AQP0-AQP12) have been identified ${ }^{[2]}$. These proteins are selectively permeated by water (aquaporins) ${ }^{[3]}$ or water plus glycerol (aquaglyceroporins) ${ }^{[4]}$. Aquaporins are strategically located at membrane sites in a variety of epithelial cells, most of which have well-defined transport functions; some are involved in fluid absorption, others in fluid secretion ${ }^{[5]}$. Aquaporin 1 (AQP1) was the first member of the AQP family to be identified, initially as a molecular component of the Colton blood group antigens in erythrocytes and subsequently cloned from kidney complementary DNA libraries and shown to possess water transporting activity ${ }^{[6,7]}$. Although AQP1 has been found to be important in osmotic water movement across cell membranes of many epithelial and endothelial barriers, its expression in the gastrointestinal system is exclusively limited to microvascular endothelia (Figure 1) and other aquaporin isoforms are known to be expressed in the epithelial cell lining. These aquaporins include $\mathrm{AQP} 3^{[8-10]}, \mathrm{AQP} 4^{[11-13]}, \mathrm{AQP} 5^{[14,15]}, \mathrm{AQP} 8^{[16,17]}, \mathrm{AQP} 9^{[18]}$ and $\mathrm{AQP} 10^{[19-22]}$. It is therefore clearly established that multiple aquaporin isoforms are present along the gastrointestinal system; some of these are classical aquaporins (i.e., AQP1, AQP4, AQP5, AQP8) and are probably mainly involved in water homeostasis ${ }^{[23]}$, whereas others are aquaglyceroporins (i.e., AQP3, AQP7, AQP9, AQP10) and are potentially involved in the facilitated movement of small uncharged organic molecules, such as glycerol ${ }^{[4]}$ as well as water. In the fluid-transporting epithelia of the kidney nephron, AQP1 is permeated by water, driven by osmotic gradients and AQP1 is abundant in the apical and basolateral membranes of renal proximal tubules and descending thin limbs and plays a key role in setting up and maintaining the countercurrent multiplication system ${ }^{[23,24]}$. Although AQP1 is known to be present in a number of extrarenal tissues, such as the ciliary body of the eye and the choroid plexus in the brain ${ }^{[6,23,25]}$, it has not been reported in normal epithelial cells lining the gastrointestinal system ${ }^{[23]}$. The presence of AQP1 in gastrointestinal epithelial cells has only been reported in tumors of the colon, where it has been reported to contribute to tumor angiogenesis and the formation of high interstitial fluid pressures and high vascular permeability of tumor microvessels ${ }^{[2]}$.

Jin $e t a l^{[1]}$ conclude that porcine AQP1 (pAQP1) is the first porcine aquaporin to be identified by means of molecular biology techniques. This is a valid and correct statement. However, the functional data they provide is from transfected $\mathrm{CHO}$ cells and red blood cells. The functional data presented by Jin $e t a^{\left[{ }^{1]}\right.}$ may not be used to support the statement that pAQP1 plays a key role in fluid transport in epithelial and endothelial structures of the pig gut. Furthermore, the immunohistochemical and Northern blot data presented by the authors do not prove that the observed differences in $\mathrm{pAQP} 1 \mathrm{mRNA}$ and protein abundance in porcine liver, small intestine, colon and salivary glands are due to differential and 


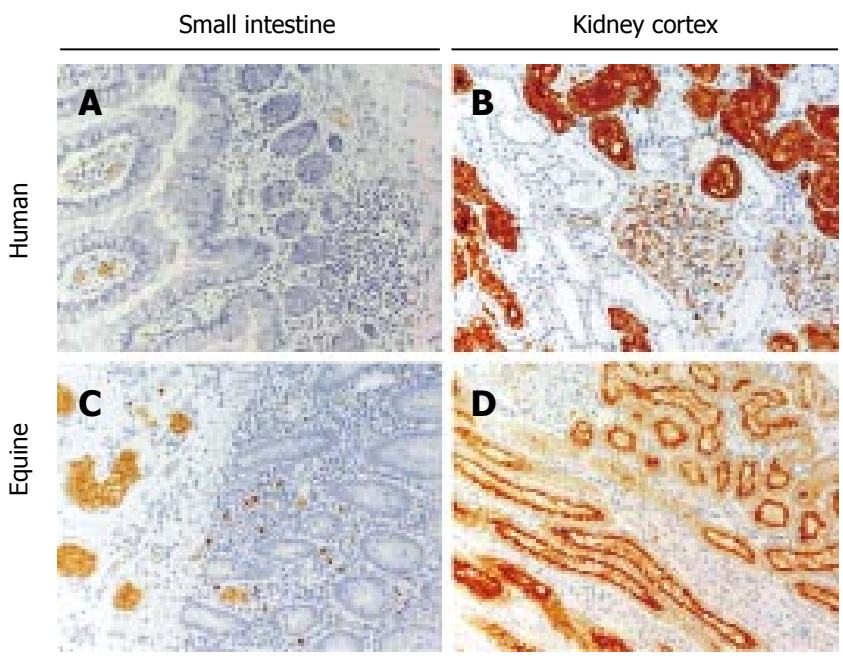

Figure $1 \mathrm{AQP} 1$ expression in human and equine small intestine and kidney. In the small intestine of both species, AQP1 is present exclusively in microvesse endothelia and in erythrocytes (panels $\mathbf{A}$ and $\mathbf{C}$ ). AQP1 is not expressed in epithelial cells or any other structures in the small intestine (panels $\mathbf{A}$ and $\mathbf{C}$ ). In the kidney of both species specific AQP1 expression is abundant in the renal cortex, specifically in the apical (brush border) and basolateral membranes of proximal tubules and in microvessel endothelia (panels B and D). Some AQP1 expression is also seen within the glomerulus in podocytes (panels $\mathbf{B}$ and $\mathbf{D}$ ).

functional expression; the differential expression observed is more likely to be the consequence of the dissimilar vascularization of these tissues which will indisputably affect the abundance of AQP1-positive endothelial cells.

Based on the evidence from studies by other investigators, it is probably premature to suggest that AQP1 is involved in fluid secretion, fluid absorption, digestive function and pathophysiology of the porcine gastrointestinal system ${ }^{[1]}$. Multiple aquaporins are likely to be involved in the processes of fluid secretion and absorption in the digestive system. Aquaporins 3, 4, 8, 9 and 10 are also known to be expressed in strategic points along the gastrointestinal system ${ }^{[13]}$. The presence of several different AQP isoforms along the gastrointestinal tract is likely to be related to the specific water-transporting functions of each of its segments. For example, salivary glands synthesize salivary amylase and mucus which are secreted along with electrolytes and water. The exocrine pancreas synthesizes proteases, lipases and amylases which are secreted with $\mathrm{HCO}_{3}^{-}$and water. The liver and gallbladder are involved in bile salt synthesis, its secretion, storage and concentration, respectively. Gastric parietal cells secrete $\mathrm{HCl}$ into the lumen of the stomach and hence require $\mathrm{AQP}$ water channels for their secretory function. Finally, the small and large intestines absorb up to $8.5 \mathrm{~L}$ and $0.4 \mathrm{~L}$ of water every day (Figure 2).

Studies on AQP-knockout mice have shown that some of the observed phenotypes are quite mild, especially in the gastrointestinal system which is not surprising given that water transport across the digestive epithelial barriers seems to occur not only via aquaporin water channels but also via other transporters, co-transporters or channel systems. The mild phenotypes of AQP-knockout mice also suggest that other aquaporins participate in compensatory mechanisms resulting from the selective disruption of a particular AQP gene.
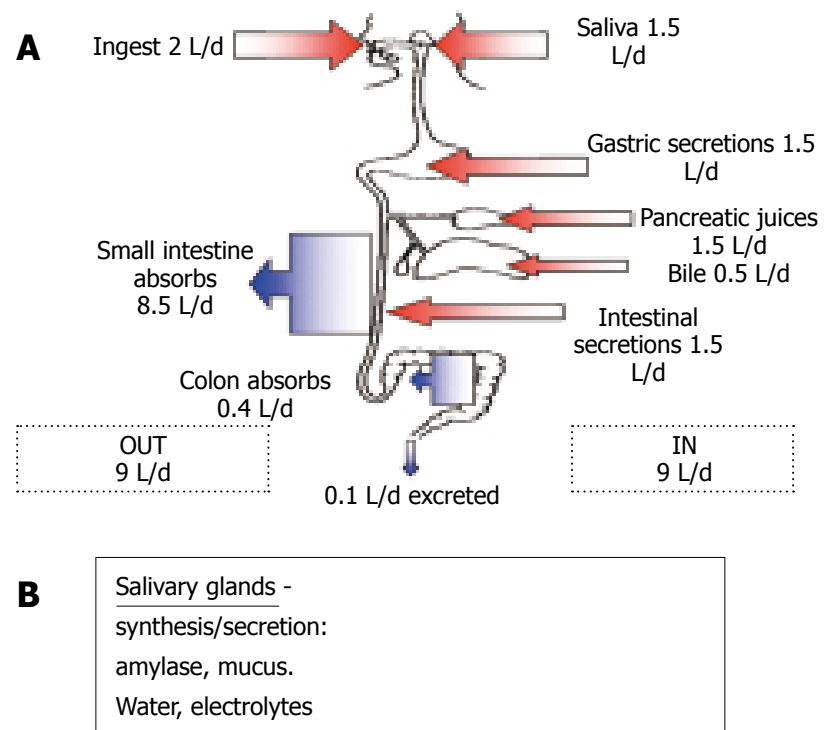

Exocrine pancreas -
synthesis/secretion:
proteases,lipase,amylase.
$\mathrm{HCO}_{3}{ }^{-}$, water

Liver -

bile salt synthesis, bile secretion.

Gall bladder -

storage and concentration of bile

Figure 2 Summary of the gastrointestinal system's daily fluid balance $(\mathrm{A})$ and the secretions of salivary glands, exocrine pancreas and liver (B).

The anatomical localization and molecular identity of AQPs in the gastrointestinal tract is not particularly well studied compared to other organs, such as the kidney. There are very few published models of water and small solute transport across the epithelium of the gut. Accordingly, the focus of future studies should be identification and subcellular localization of AQP proteins in the mammalian (human) gut. We have recently proposed a model (Figure 3) of water and small solute transport across the epithelium of the human gut which incorporates the functional contribution of AQP1, AQP3, AQP8 and AQP10 ${ }^{[21]}$. The data presented by Jin et a ${ }^{[1]}$ also confirm our findings ${ }^{[23]}$ and the findings of several other studies with regard to the distribution of the AQP1 protein in microvessel endothelia and bile ducts. However, further studies are still required to determine the expression of the recently identified members of the AQP family in the gastrointestinal system of humans in health and in diseases brought about by opportunistic bacteria (i.e., diarrhoea, colic and endotoxemia), immune disorders of the gut, such as Crohn's disease and cancers of gastrointestinal organs. From a biological viewpoint, it would be of interest to perform comparative studies of AQP expression and function in gut tissues derived from mammals, amphibians, reptiles and fish. Also, determining the expression of AQP proteins in guts of omnivores, herbivores and carnivores will help us gain a more complete understanding of the functions of these proteins in the mammalian gastrointestinal system. 


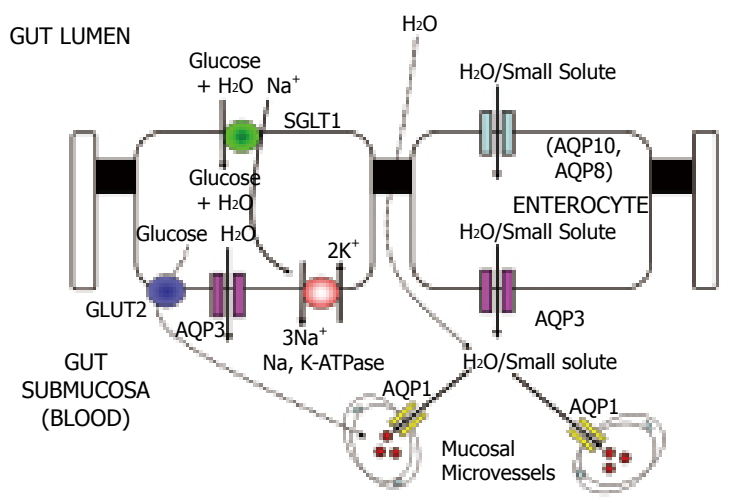

Figure 3 Model of water and small solute transport across the absorptive epithelium of the human small intestine ${ }^{[21]}$. The potential routes for water entry into the enterocyte from the luminal (apical) side are AQP10 and the sodium/glucose co-transporter SGLT1. SGLT1 is a secondary active transporter driven by the function of basolateral $\mathrm{Na}, \mathrm{K}-\mathrm{ATP}$ ase which co-transports $\mathrm{Na}+$ and glucose along with water into enterocytes. AQP8 (shown in parentheses) has been shown to be located in the sub-apical cytoplasm of absorptive epithelial cells. The basolateral water exit pathway is most likely to be the AQP3 protein ${ }^{[10,28]}$. Although the location of the AQP7 protein has not yet been precisely determined, it may be involved in glycerol transport in the intestinal lacteal system.

\section{REFERENCES}

1 Jin SY, Liu YL, Xu LN, Jiang Y, Wang Y, Yang BX, Yang H, Ma TH. Cloning and characterization of porcine aquaporin 1 water channel expressed extensively in gastrointestinal system. World J Gastroenterol 2006; 12: 1092-1097

2 Borgnia M, Nielsen S, Engel A, Agre P. Cellular and molecular biology of the aquaporin water channels. Annu Rev Biochem 1999; 68: 425-458

3 Agre $\mathbf{P}$, Nielsen $\mathrm{S}$. The aquaporin family of water channels in kidney. Nephrologie 1996; 17: 409-415

4 Engel A, Stahlberg H. Aquaglyceroporins: channel proteins with a conserved core, multiple functions, and variable surfaces. Int Rev Cytol 2002; 215: 75-104

5 Brown D, Katsura T, Kawashima M, Verkman AS, Sabolic I. Cellular distribution of the aquaporins: a family of water channel proteins. Histochem Cell Biol 1995; 104: 1-9

6 Agre P, Preston GM, Smith BL, Jung JS, Raina S, Moon C, Guggino WB, Nielsen S. Aquaporin CHIP: the archetypal molecular water channel. Am J Physiol 1993; 265: F463-F476

7 Agre $\mathbf{P}$, Smith BL, Preston GM. ABH and Colton blood group antigens on aquaporin-1, the human red cell water channel protein. Transfus Clin Biol 1995; 2: 303-308

8 Takata K, Matsuzaki T, Tajika Y. Aquaporins: water channel proteins of the cell membrane. Prog Histochem Cytochem 2004; 39: 1-83

9 Matsuzaki T, Tajika Y, Ablimit A, Aoki T, Hagiwara H, Takata K. Aquaporins in the digestive system. Med Electron Microsc 2004; 37: 71-80

10 Mobasheri A, Wray S, Marples D. Distribution of AQP2 and AQP3 water channels in human tissue microarrays. J Mol Histol 2005; 36: 1-14

11 Ma T, Yang B, Verkman AS. Cloning of a novel water and urea-permeable aquaporin from mouse expressed strongly in colon, placenta, liver, and heart. Biochem Biophys Res Commun
1997; 240: 324-328

12 Koyama Y, Yamamoto T, Tani T, Nihei K, Kondo D, Funaki H, Yaoita E, Kawasaki K, Sato N, Hatakeyama K, Kihara I. Expression and localization of aquaporins in rat gastrointestinal tract. Am J Physiol 1999; 276: C621-C627

13 Ma T, Verkman AS. Aquaporin water channels in gastrointestinal physiology. J Physiol 1999; 517 ( Pt 2): 317-326

14 Matsuzaki T, Tajika Y, Suzuki T, Aoki T, Hagiwara H, Takata $\mathrm{K}$. Immunolocalization of the water channel, aquaporin-5 (AQP5), in the rat digestive system. Arch Histol Cytol 2003; 66: 307-315

15 Parvin MN, Tsumura K, Akamatsu T, Kanamori N, Hosoi K. Expression and localization of AQP5 in the stomach and duodenum of the rat. Biochim Biophys Acta 2002; 1542: 116-124

16 Calamita G, Mazzone A, Bizzoca A, Cavalier A, Cassano G, Thomas D, Svelto M. Expression and immunolocalization of the aquaporin-8 water channel in rat gastrointestinal tract. Eur J Cell Biol 2001; 80: 711-719

17 Elkjaer ML, Nejsum LN, Gresz V, Kwon TH, Jensen UB, Frøkiaer J, Nielsen S. Immunolocalization of aquaporin-8 in rat kidney, gastrointestinal tract, testis, and airways. Am J Physiol Renal Physiol 2001; 281: F1047-F1057

18 Okada S, Misaka T, Matsumoto I, Watanabe H, Abe K. Aquaporin-9 is expressed in a mucus-secreting goblet cell subset in the small intestine. FEBS Lett 2003; 540: 157-162

19 Hatakeyama S, Yoshida Y, Tani T, Koyama Y, Nihei K, Ohshiro K, Kamiie JI, Yaoita E, Suda T, Hatakeyama K, Yamamoto $\mathrm{T}$. Cloning of a new aquaporin (AQP10) abundantly expressed in duodenum and jejunum. Biochem Biophys Res Commun 2001; 287: 814-819

20 Ishibashi K, Morinaga T, Kuwahara M, Sasaki S, Imai M. Cloning and identification of a new member of water channel (AQP10) as an aquaglyceroporin. Biochim Biophys Acta 2002; 1576: $335-340$

21 Mobasheri A, Shakibaei M, Marples D. Immunohistochemical localization of aquaporin 10 in the apical membranes of the human ileum: a potential pathway for luminal water and small solute absorption. Histochem Cell Biol 2004; 121: 463-471

22 Li H, Kamiie J, Morishita Y, Yoshida Y, Yaoita E, Ishibashi K, Yamamoto T. Expression and localization of two isoforms of AQP10 in human small intestine. Biol Cell 2005; 97: 823-829

23 Mobasheri A, Marples D. Expression of the AQP-1 water channel in normal human tissues: a semiquantitative study using tissue microarray technology. Am J Physiol Cell Physiol 2004; 286: C529-C537

24 Pallone TL, Turner MR, Edwards A, Jamison RL. Countercurrent exchange in the renal medulla. Am J Physiol Regul Integr Comp Physiol 2003; 284: R1153-R1175

25 Speake T, Freeman LJ, Brown PD. Expression of aquaporin 1 and aquaporin 4 water channels in rat choroid plexus. Biochim Biophys Acta 2003; 1609: 80-86

26 Mobasheri A, Airley R, Hewitt SM, Marples D. Heterogeneous expression of the aquaporin 1 (AQP1) water channel in tumors of the prostate, breast, ovary, colon and lung: a study using high density multiple human tumor tissue microarrays. Int J Oncol 2005; 26: 1149-1158

27 Ramírez-Lorca R, Vizuete ML, Venero JL, Revuelta M, Cano J, Ilundáin AA, Echevarría M. Localization of aquaporin-3 mRNA and protein along the gastrointestinal tract of Wistar rats. Pflugers Arch 1999; 438: 94-100 